\title{
Prebiotic Driven Increases in IL-17A Do Not Prevent Campylobacter jejuni Colonization of Chickens
}

\author{
Geraldine M. Flaujac Lafontaine ${ }^{1}$, Philip J. Richards', Phillippa L. Connerton ${ }^{1}$, \\ Peter M. O'Kane', Nacheervan M. Ghaffar', Nicola J. Cummings ${ }^{1}$, Neville M. Fish ${ }^{2}$ and \\ lan F. Connerton ${ }^{1 *}$
}

' Division of Microbiology, Brewing and Biotechnology, School of Biosciences, University of Nottingham, Loughborough, United Kingdom, ${ }^{2}$ Saputo Dairy UK, Dairy Crest Innovation Centre, Harper Adams University, Newport, United Kingdom

Worldwide Campylobacter jejuni is a leading cause of foodborne disease. Contamination of chicken meat with digesta from $C$. jejuni-positive birds during slaughter and processing is a key route of transmission to humans through the food chain. Colonization of chickens with $C$. jejuni elicits host innate immune responses that may be modulated by dietary additives to provide a reduction in the number of campylobacters colonizing the gastrointestinal tract and thereby reduce the likelihood of human exposure to an infectious dose. Here we report the effects of prebiotic galacto-oligosaccharide (GOS) on broiler chickens colonized with C. jejuni when challenged at either an early stage in development at 6 days of age or 20 days old when campylobacters are frequently detected in commercial flocks. GOS-fed birds had increased growth performance, but the levels of $C$. jejuni colonizing the cecal pouches were unchanged irrespective of the age of challenge. Dietary GOS modulated the immune response to $C$. jejuni by increasing cytokine IL-17A expression at colonization. Correspondingly, reduced diversity of the cecal microbiota was associated with Campylobacter colonization in GOS-fed birds. In birds challenged at 6 days-old the reduction in microbial diversity was accompanied by an increase in the relative abundance of Escherichia spp. Whilst immuno-modulation of the Th17 pro-inflammatory response did not prevent $C$. jejuni colonization of the intestinal tract of broiler chickens, the study highlights the potential for combinations of prebiotics, and specific competitors (synbiotics) to engage with the host innate immunity to reduce pathogen burdens.

Keywords: Campylobacter, galacto-oligosaccharide, prebiotic, broiler chicken, innate immunity, microbiota, Th17, pro-inflammatory response

Received: 23 September 2019 Accepted: 17 December 2019

Published: 14 January 2020

Citation:

Flaujac Lafontaine GM, Richards PJ, Connerton PL, O'Kane PM, Ghaffar NM, Cummings NJ, Fish NM and Connerton IF (2020) Prebiotic Driven Increases in IL-17A Do Not Prevent Campylobacter jejuni Colonization of Chickens.

Front. Microbiol. 10:3030 doi: 10.3389/fmicb.2019.03030

\section{INTRODUCTION}

Campylobacter spp. are recognized as the major contributor to bacterial foodborne illness worldwide (Kaakoush et al., 2015). Campylobacterosis was the most frequently reported human zoonotic disease in the European Union in 2017 with 246,158 confirmed cases of gastrointestinal illness (EFSA, 2018). The most common species associated with human disease is C. jejuni (84.4\%), but C. coli also represent a significant disease burden (9.2\%; EFSA, 2018). C. jejuni and

Abbreviations: C. jejuni, Campylobacter jejuni; CFU, colony forming unit; FCR, feed conversion ratio; GoI, gene of interest; GOS, galacto-oligosaccharide; GIT, gastro-intestinal tract; IL, interleukin. 
C. coli are referred to as thermophilic species as they can grow at $42^{\circ} \mathrm{C}$, making them suited to colonize the intestinal tracts of poultry species (reviewed by Sahin et al., 2015). Poultry are a major source of campylobacters with an estimated $80 \%$ of human illness arising from poultry sources (Andreoletti et al., 2010). Source attribution estimates referenced at the point of exposure indicate $65-69 \%$ of human cases are from exposure to chicken meat (Ravel et al., 2017). Poultry meat is frequently contaminated with intestinal content harboring high levels of Campylobacter cells during slaughter and carcass processing, which constitutes the main risk to public health (Osimani et al., 2017). This has prompted the EU to adopt a microbiological sampling plan for broiler chicken carcasses with a limit of 1,000 CFU/g (Commission Regulation (EU) 2017/1495). Strict on-farm biosecurity measures to prevent Campylobacter exposure and flock colonization of broiler chickens have been implemented in many countries, but these alone do not maintain Campylobacter-free flocks (Newell et al., 2011). Intervention strategies have been developed aimed at reducing levels of Campylobacter colonization, and thereby human exposure, if the reductions can be translated on to chicken meat (Rosenquist et al., 2003; Newell et al., 2011; Sahin et al., 2015). Campylobacter colonization has been associated with poor flock health and performance in commercial broiler chicken production (Bull et al., 2008), although performance issues are not manifest in all circumstances (Gormley et al., 2014). The impact of Campylobacter colonization on bird health has been reported to vary with the broiler breed/rate of growth, stocking density, intercurrent infectious or immunosuppressive challenges and the colonizing organism (Humphrey et al., 2015; Li L. et al., 2018). It is, however, clear that Campylobacter colonization elicits a Th17 pro-inflammatory response in broiler chickens (Reid et al., 2016; Connerton et al., 2018). Intestinal intraepithelial lymphocytes characterized as either $\mathrm{CD}^{+} \mathrm{CD} 25^{+}$cells or $\gamma \delta$ $\mathrm{T}$ cells have been reported to express intracellular IL-17A in the lower intestine of chickens (Walliser and Göbel, 2018). Not all inflammatory responses lead to negative outcomes for the host. It has been questioned whether dietary anti-inflammatory additives aimed at the detrimental consequences of intestinal inflammation may actually impair necessary responses of young animals that are required to overcome the challenges present in commercial production to achieve favorable performance outcomes (Broom and Kogut, 2018). Zootechnical performance remains a key driver in the poultry industry, at the same time public and regulatory concerns are mounting regarding welfare and antibiotic use in poultry production. Although progress has been made toward reducing antibiotic use in poultry production in several countries has been reported more remains to be achieved (Speksnijder et al., 2015). It has been proposed that the rational manipulation of poultry feed formulation can improve pathogen resistance, improve production, and reduce the threat posed by zoonotic pathogens through the food chain (Kogut, 2009; Swaggerty et al., 2019). Intestinal innate immune responses to feed and pathogen challenges are strongly influenced by the gut microbiota (Kogut et al., 2018). The addition of probiotic microorganisms, prebiotics and phytobiotics in feed are approaches by which the gut microbiota of broiler chickens may be influenced (reviewed by Pedroso et al., 2013; Pourabedin and Zhao, 2015; Van Immerseel et al., 2017; Clavijo and Flórez, 2018). It is proposed that these approaches will be most effective when introduced early in life to establish a robust microbiota that benefits production (Rubio, 2019). We have recently reported that the inclusion of the prebiotic GOS in juvenile broiler feed enhances the growth and feed conversion rates of broiler chickens, increases ileal and cecal IL-17A gene expression and brings about changes in the cecal populations of key Lactobacillus spp. (Richards et al., 2019b). GOS represent host-indigestible carbohydrates that have been identified as promoting beneficial bacteria in humans and animals, which include Bifidobacteria, Bacteroides, and Lactobacillaceae (Jung et al., 2008; Hughes et al., 2017; Van Bueren et al., 2017; Tian et al., 2019).

In the present study, we have examined whether the impact of a GOS diet on host fitness, immune response and changes in the gut microbiota would support the clearance of Campylobacter jejuni in broiler chickens. For this purpose, we fed isocaloric GOS or control diets from hatch to 20 days of age to modulate the intestinal innate immune status and gut microbiota of broiler chickens. Two approaches were taken to determine the role of development on host response. In one experiment birds were challenged at an early stage of development at 6 days old to determine the persistence of $C$. jejuni in the modified gut environment and assess corresponding intestinal chemokine and cytokine gene expression, and the prevailing intestinal microbiota throughout the typical broiler chicken lifespan of 35 days. In a separate experiment birds were challenged at 20 days old, when campylobacters are frequently first detected in commercial flocks, with similar observations made until the trial ended when birds reached 35 days old.

\section{MATERIALS AND METHODS}

\section{Trial Design}

Two independently performed trials monitored the effect of dietary GOS on development of the gut innate immune responses and the cecal microbiota of broiler chickens challenged with C. jejuni HPC5 at either an early stage of development (6 days old) or late stage (20 days old), the age at which birds often become Campylobacter positive in commercial production. Birds were randomly assigned to either a group fed a control diet (referred to as Campylobacter) or to a group fed a GOSsupplemented diet (referred to as GOS + Campylobacter) for the duration of the experiment. In the early 6 -day old challenge experiment (referred to as 6-dc), two groups of 35 birds were kept in pens from day of hatch until day 6 when all birds were administered C. jejuni, and subsequently independently caged until the end of the study on day 35 . Birds were randomly selected $(n=7)$ from each diet group and euthanized prior to sampling intestinal tissues and contents at $8,15,22,28$, and 35 days of age (da). For the late 20-day challenge trial (referred to as 20-dc), two groups of 21 birds were similarly housed in pens until 20 days when the birds were administered C. jejuni and independently caged until the end of the study at 35 days. Again, randomly selected birds $(n=7)$ from each diet group were euthanized 
for intestinal sampling at 22, 28, and 35 days. All experimental birds post challenge were maintained in independent housing to prevent the birds sharing intestinal microbiota through coprophagy, which would otherwise confound the experimental design by reducing the number of replicates.

\section{Experimental Animals}

Day-of-hatch male Ross 308 broiler chicks purchased from a local hatchery were randomly assigned on the basis of weight to control or GOS diet groups. Birds were brooded in floor pens on wood shavings until the day of Campylobacter challenge. Birds were housed in a controlled environment under strict conditions of biosecurity and kept under controlled light (L:D 12:12) with ad libitum access to food and water throughout the study. Temperatures conformed to the Code of Practice for the Housing and Care of Animals Bred, Supplied or Used for Scientific Purposes 2014. Welfare monitoring of the chickens was undertaken two or three times every $24 \mathrm{~h}$ post Campylobacter challenge. Birds in the control group were sustained on a wheat-based diet provided as starter crumb for 0-10 days, grower pellets for 11-24 days and finisher pellets for 2535 days. The starter diet contained wheat $(59.9 \% \mathrm{w} / \mathrm{w})$, soya meal $(32.5 \% \mathrm{w} / \mathrm{w})$, soybean oil $(3.65 \% \mathrm{w} / \mathrm{w})$, limestone $(0.6 \%$ $(\mathrm{w} / \mathrm{w})$, calcium phosphate $(1.59 \% \mathrm{w} / \mathrm{w})$, sodium bicarbonate $(0.27 \% \mathrm{w} / \mathrm{w})$, the enzymes phytase and xylanase (dosed according to the manufacturer's instructions; DSM Nutritional Products Ltd., PO Box 2676 CH-4002 Basel, CH) and a vitamin mix containing $\mathrm{NaCl}$ salt, lysine $\mathrm{HCl}$, DL-methionine and threonine. The grower and finisher diets increased the wheat content at the expense of soya meal by 2 and $5 \% \mathrm{w} / \mathrm{w}$, respectively. The 6-dc prebiotic GOS + Campylobacter treatment group had the starter feed supplemented with GOS from 1 to 10 days at $3.37 \% \mathrm{w} / \mathrm{w}$ and then $11-35$ days at $1.695 \% \mathrm{w} / \mathrm{w}$, whilst the $20-\mathrm{dc}$ birds were fed $3.37 \%$ w/w GOS throughout the experiment. The GOS was provided as Nutrabiotic ${ }^{\circledR}$ GOS that contains $74 \%$ GOS w/w dry matter (Dairy Crest Ltd., Davidstow, Cornwall, United Kingdom). GOS preparations contain a mixture of monosaccharides (glucose and galactose) and oligosaccharides (DP2 - DP8) with the exception of lactose that is a residual component of the production process. The enzymatic synthesis of GOS produces $\beta-(1-3)$ or $\beta-(1-4)$ or $\beta$-(1-6)-linked galactose residues (1 to 7 ) with a terminal $\beta$ $(1-3)$ or $\beta-(1-4)$ or $\beta-(1-6)$-linked glucose. Isocaloric content adjustments for GOS inclusion were for the starter feed (wheat $54.0 \% \mathrm{w} / \mathrm{w})$ soya meal $(33.9 \% \mathrm{w} / \mathrm{w})$ and soybean oil $(4.88 \% \mathrm{w} / \mathrm{w})$; for the grower feed (wheat $54.7 \% \mathrm{w} / \mathrm{w}$ ) soya meal $(32.2 \% \mathrm{w} / \mathrm{w})$ and soybean oil $(6.76 \% \mathrm{w} / \mathrm{w})$; for the finisher feed (wheat $60.33 \% \mathrm{w} / \mathrm{w})$ soya meal $(26.7 \% \mathrm{w} / \mathrm{w})$ and soybean oil $(6.84 \% \mathrm{w} / \mathrm{w})$. The feed and paper liners on which the chicks were delivered were found negative for Salmonella using standard enrichment procedures. At the time of challenge all birds were administered by oral gavage a dose of $1 \times 10^{7} \mathrm{CFU}$ C. jejuni HPC5, a well-characterized broiler chicken isolate, suspended in MRD (Oxoid, Thermo Fisher Scientific, Altrincham, United Kingdom) in volumes of $0.1 \mathrm{ml}$ for the 6 -dc birds or in $1 \mathrm{ml}$ for the $20-\mathrm{dc}$ birds. For sample collection, birds were euthanized by either exposure to rising $\mathrm{CO}_{2}$ gas or parenteral barbiturate overdose followed by cervical dislocation depending on bird mass in accordance with Schedule 1 of the United Kingdom Animals (Scientific Procedures) Act 1986. Ileal tissues were collected from approximately $3 \mathrm{~cm}$ distal to Meckel's diverticulum and cecal tissues isolated from the distal tips of the cecal pouches. Samples of intestinal tissue were immediately frozen in liquid nitrogen for subsequent RNA isolation or preserved in $10 \%$ $(\mathrm{w} / \mathrm{v})$ neutral buffered formalin (Thermo Fisher Scientific) for histological assessment. Cecal contents were collected and used either to enumerate Campylobacter or for isolation of total genomic DNA extraction.

\section{Performance and Growth Rate}

Live weights and all feed consumed were recorded for all birds at regular intervals from the start of the experiment until the end at 35 days. FCR were calculated as a ratio of feed consumed to the live weight of the birds. Bird growth rates were compared for each of the birds that remained at the end of the 35 days rearing period that collectively represent all the birds for which repeated measurements of the mass were recorded throughout the broiler chicken lifespan.

\section{Bacterial Enumeration}

Approximately $1 \mathrm{~g}$ of digesta was aseptically collected from both ceca and combined in pre-weighed universal containers before a $10 \% \mathrm{w} / \mathrm{v}$ suspension was prepared in MRD. Campylobacter were enumerated in triplicate from decimal dilutions prepared in MRD using a modification of the Miles and Misra technique (Miles et al., 1938). For each triplicate dilution set, five aliquots were dispensed onto CCDA agar (PO0119; Oxoid) prepared with the addition of agar to $2 \%$ (to prevent swarming) and with addition of CCDA Selective Supplement SR0155 (Oxoid). Plates were incubated at $42^{\circ} \mathrm{C}$ in a microaerobic atmosphere $\left(2 \% \mathrm{H}_{2}\right.$, $5 \% \mathrm{CO}_{2}, 5 \% \mathrm{O}_{2}$, and $88 \% \mathrm{~N}_{2} \mathrm{v} / \mathrm{v}$ ) for $48 \mathrm{~h}$ (Don Whitley Scientific modified atmospheric cabinet, Shipley, United Kingdom).

\section{Histology}

Tissue samples fixed in a $10 \%$ formalin solution were dehydrated through a series of alcohol solutions, cleared in xylene, and embedded in paraffin wax (Microtechnical Services Ltd., Exeter, United Kingdom). Sections (3 to $5 \mu \mathrm{m}$ thick) were prepared and stained with modified hematoxylin and eosin (H\&E). After staining, the slides were scanned by NanoZoomer Digital Pathology System (Hamamatsu, Welwyn Garden City, United Kingdom). Villus height and crypt depth were recorded from operator blinded measurements collected using the NanoZoomer Digital Pathology Image Program (Hamamatsu) from histology stained slides scanned at $40 \times$ resolution for each tissue sample. Villus height was determined from the tip of the villus to the crypt opening and the associated crypt depth was measured from the base of the crypt to the level of the crypt opening. The ratios of villus height to relative crypt depth (v/c ratio) were calculated from these measurements. Dimensions for 10 well-oriented villi per tissue sample of 3 or 4 birds per diet group at each sampling time were analyzed. 


\section{RNA Isolation and RT-qPCR of the Cytokines and Chemokines}

Total RNAs were isolated from ceca and ileum tissue biopsies using NucleoSpin RNA purification kit (Macherey-Nagel, GmbH \& co. KG, Düren; DE) according to the manufacturer's protocol with the following modifications. Tissue samples were homogenized with the kit Lysis buffer and $2.8 \mathrm{~mm}$ ceramic beads (MO BIO Laboratories Inc., Carlsbad, United States) using TissueLyser II (Qiagen, Hilden, Germany). Subsequently total RNAs were extracted as described in the protocol with a DNaseI treatment step as per the manufacturer's instructions. Purified RNAs were eluted in nuclease free water, validated for quality and quantity using UV spectrophotometry (Nanodrop ND-1000, Labtech International Ltd., Uckfield, United Kingdom), and stored long term at $-80^{\circ} \mathrm{C}$. RNAs with OD260/280 ratio between 1.9 and 2.1 were deemed high quality, the ratios were found with a mean of $2.12 \pm 0.01$. Reverse Transcription was performed with $1 \mu \mathrm{g}$ of RNA, SuperScript II (Invitrogen Life Technologies, Carlsbad, CA, United States) and random hexamers as described previously (Connerton et al., 2018). Quantitative PCR reaction was performed with cDNA template derived from $4 \mathrm{ng}$ of total RNA in triplicate using SYBR Green Master mix (Applied Biosystems, Thermo Fisher Scientific). The RNA level of expression was determined by qPCR using the Roche Diagnostics LightCycler 480 (Hoffmann La Roche AG, CH). The primers sequence for GAPDH, INF- $\gamma$, IL-1 $\beta$, IL-6, IL-10, IL-17A, IL17F, ChCXCLi1, and ChCXCLi2 (Table 1) were previously described (Kaiser et al., 2003; Nang et al., 2011; Rasoli et al., 2015; Reid et al., 2016). Cytokines and chemokines transcripts fold change (FC) were calculated according to the manufacturer using the $2^{-\Delta \Delta C p}$ method (Livak and Schmittgen, 2001). Averages of the triplicate $\mathrm{Ct}$ values were analyzed with the target genes of interest (GOI) values normalized to those of the housekeeping gene Glyceraldehyde 3-phosphate dehydrogenase (GAPDH).

\section{Microbiota Analysis}

DNA was isolated from cecal content using the MoBio PowerSoil kit (now QIAGEN Ltd., Manchester, United Kingdom) according to the manufacturer's instructions. The V4 regions of the bacterial 16S rRNA genes were PCR amplified using the primers 515f (5' GTGCCAGCMGCCGCGGTAA $\left.3^{\prime}\right)$ and 806r (5' GGACTACHVGGGTWTCTAAT 3') (Caporaso et al., 2011). Amplicons were then sequenced on the Illumina MiSeq platform (Illumina Inc., San Diego, United States) using $2 \times 250$ bp cycles (reagent kit V2). The 16S rRNA gene sequences were quality filtered and clustered into OTUs in Mothur (Schloss et al., 2009) using the Schloss lab. MiSeq SOP $^{1}$, accessed 2018-10-05; Kozich et al., 2013). Batch files of Mothur commands used in this study are available at https: //github.com/PJRichards/lafontaine_campy_gos. Raw sequences for $16 \mathrm{~S}$ rDNA data originally reported in this article are deposited in the NCBI database within BioProject PRJNA380214

${ }^{1}$ https://github.com/mothur/mothur under SRA study SRP133552. Comparative 16S rDNA data from mock-challenged birds reproduced in this study was downloaded from NCBI PRJNA380214 (the FTP code is available from GitHub repository as described below). Postprocessing rarefaction curves were plotted to assess sampling effort (Supplementary Figure S1).

\section{Data and Statistical Analysis}

All figures were drawn and unless otherwise stated all tests for statistical significance were performed using R 3.6.1 (R Core Team, 2019) in RStudio 1.2.1 (RStudio Team, 2015). All R scripts have been made available here: https://github.com/PJRichards/ lafontaine_campy_gos. Histology measurements for each diet regimen of age-matched birds were compared using ANOVA.

\section{Zootechnical Data}

Bird growth rates were compared by determining rate of growth from 15 days for each of the birds that remained at the end of the trial at 35 days, i.e., birds for which repeated measurements of the mass were recorded throughout the growing period (Campylobacter treatment group, $n=7$; GOS + Campylobacter treatment, $n=8)$. Growth rate was determined for individual birds for the period of linear growth post-challenge (6-dc birds between 15 and 35 days; 20 -dc birds between 22 and 35 days). Growth rates (g/day) were compared between cohorts using Student's $t$ test. Further comparison was made between the mass of age-matched birds using Student's $t$ test. C. jejuni viable counts were $\log _{10}$-transformed and tested for significance using Student's $t$ test.

\section{Microbiota - 16S rRNA Gene Sequence Data}

Comparisons were made of $\alpha$-diversity metrics (Shannon diversity and inverse Simpson's indices) generated in mothur. For the four treatment groups in 6 -dc birds at 8 days ( $2 \mathrm{dpi}$ ) differences in $\alpha$-diversity were tested for using ANOVA with Tukey multiple comparison of means test ( $p$ was adjusted for multiple comparisons). For subsequent comparisons of $\alpha$-diversity at 15,22, 28 and 35 days between two treatment groups only (Campylobacter and GOS + Campylobacter) Student's $t$ test was used to test for significance. Correspondingly, comparisons of Chao richness between the four treatment groups in 6-dc birds at 8 days $(2 \mathrm{dpi})$ were made using a Kruskal-Wallis test with Benjamini-Hochberg FDR correction as were subsequent comparisons at $15,22,28$, and 35 days. Note that a randomly selected community was deleted from the GOS + Campylobacter treatment group at 15 days and 35 (birds 2 and 6, respectively) to even group size and allow unbiased comparisons. For 20-dc birds, comparisons of $\alpha$-diversity were made using Student's $t$ test and comparisons of Chao richness were made using a Kruskal-Wallis test. Differences in bacterial composition were tested for by modeling compositional population data in terms of a Dirichlet distribution and using a likelihood ratio test in DirtyGenes (Shaw et al., 2019). Differential OTUs were identified with LEfSE in mothur (Schloss et al., 2009; Segata et al., 2011). 
TABLE 1 | Primer sequences for the gene expression determined by qPCR.

\begin{tabular}{|c|c|c|c|c|}
\hline Target gene & Primer sequence $\left(5^{\prime}-3^{\prime}\right)$ & Product size (bp) & NCBI Accession number & References \\
\hline GAPDH & $\begin{array}{l}\text { F: GACGTGCAGCAGGAACACTA } \\
\text { R: TCTCCATGGTGGTGA AGACA }\end{array}$ & 343 & NM_204305.1 & Nang et al. (2011) \\
\hline INF- $\gamma$ & $\begin{array}{l}\text { F: TGAGCCAGATTGTTCGATG } \\
\text { R: CTTGGCCAGGTCCATGATA }\end{array}$ & 152 & NM_205149.1 & Nang et al. (2011) \\
\hline $\mathrm{IL}-1 \beta$ & $\begin{array}{l}\text { F: GGATTCTGAGCACACCACAGT } \\
\text { R: TCTGGTTGATGTCGAAGATGTC }\end{array}$ & 272 & NM_204524.1 & Nang et al. (2011) \\
\hline IL-10 & $\begin{array}{l}\text { F: GCTGCGCTTCTACACAGATG } \\
\text { R: TCCCGTTCTCATCCATCTTC }\end{array}$ & 203 & NM_001004414.2 & Nang et al. (2011) \\
\hline IL-6 & $\begin{array}{l}\text { F: GCTCGCCGGCTTCGA } \\
\text { R: GGTAGGTCTGAAAGGCGAACAG }\end{array}$ & 71 & NM_204628.1 & Kaiser et al. (2003) \\
\hline IL-17A & $\begin{array}{l}\text { F: CATGGGATTACAGGATCGATGA } \\
\text { R: GCGGCACTGGGCATCA }\end{array}$ & 68 & NM_204460.1 & Reid et al. (2016) \\
\hline $\mathrm{IL}-17 \mathrm{~F}$ & $\begin{array}{l}\text { F: TGACCCTGCCTCTAGGATGATC } \\
\text { R: GGGTCCTCATCGAGCCTGTA }\end{array}$ & 78 & XM_426223.5 & Reid et al. (2016) \\
\hline ChCXCLi-1 & $\begin{array}{l}\text { F: CCGATGCCAGTGCATAGAG } \\
\text { R: CCTTGTCCAGAATTGCCTTG }\end{array}$ & 191 & NM_205018.1 & Rasoli et al. (2015) \\
\hline ChCXCLi-2 & $\begin{array}{l}\text { F: CCTGGTITCAGCTGCTCTGT } \\
\text { R: GCGTCAGCTTCACATCTTGA }\end{array}$ & 128 & NM_205498.1 & Rasoli et al. (2015) \\
\hline
\end{tabular}

\section{Intestinal Cytokine and Chemokine Transcription}

Host cytokine and chemokine transcript levels were assessed by RT-qPCR of transcribed RNA isolated from ileal and cecal tissue sections. Cytokine and chemokine normalized expression was determined for each sample as $2^{-\Delta} C p$ with $\Delta \mathrm{Cp}=\mathrm{Cp}$ of $\mathrm{GoI}-\mathrm{Cp}$ of housekeeping gene (GAPDH). The relative gene expression between birds fed a control (Campylobacter) or a GOS diet (GOS + Campylobacter), results were determined as a group mean FC, which was calculated from $2^{-\Delta} \Delta C p$ with $\Delta \Delta \mathrm{Cp}=\Delta \mathrm{Cp}$ (GOS diet) $-\Delta \mathrm{Cp}$ (average $\Delta \mathrm{Cp}$ of control). Differences between treatment groups were assessed using Wilcoxon rank sum tests with BenjaminiHochberg FDR correction.

\section{RESULTS}

\section{Dietary Galacto-Oligosaccharide Improved the Growth Performance of Campylobacter jejuni-Colonized Broiler Chickens}

The aim of this research was to determine whether GOS could still act as a prebiotic and confer a growth performance advantages to broiler chickens colonized when colonized by $C$. jejuni. Chickens fed a GOS diet performed better than those fed the calorie-matched control diet (Figures 1A,B). Differences in the body weights were evident from 22 days for the 6-dc birds $(p \leq 0.029)$ until slaughter at 35 days (mean body weights Campylobacter treatment $=2276 \mathrm{~g}$, GOS + Campylobacter treatment $=2722 ; p=0.029)$ (Figure 1A). Differences in the weights of the 20-da challenged birds were observed at 35 days (mean body weights Campylobacter $=2055 \mathrm{~g}$, GOS + Campylobacter $=2341 ; p=0.004)($ Figure 1B). The growth rates of the 6-da challenged birds fed GOS increased in the period 15-35 days compared to the challenged chickens fed a control diet (control = mean $83.6 \mathrm{~g} /$ day, $\mathrm{GOS}=$ mean $105.1 \mathrm{~g} / \mathrm{day} ; p=0.0233$ ), and similarly for the 20 -da challenged birds for the period 22-35 days (control = mean $85.3 \mathrm{~g} /$ day, $\mathrm{GOS}=$ mean $97.6 \mathrm{~g} /$ day; $p=0.007$ ).

The cumulative FCR up to 35 days for 6-dc birds fed control diet $(n=7)$ was 1.45 and for the GOS diet $(n=8)$ was 1.42 while the FCR for 20 -dc birds fed control diet $(n=7)$ was 1.56 and for GOS diet $(n=7)$ was 1.58 (data not shown). The contemporary breed performance objectives for male Ross 308 were body weight 2,283 g and FCR of 1.54 at 35 days (Aviagen Performance Objectives, 2014).

\section{Dietary Galacto-Oligosaccharide Inclusion Did Not Prevent Campylobacter jejuni Colonization of Broiler Chickens}

To assess the impact of dietary GOS on C. jejuni colonization we sacrificed birds over the rearing period to determine the cecal viable Campylobacter counts $(n=7)$. All birds were culturenegative for Campylobacter spp. until oral gavage with C. jejuni HPC5 at 6 days for the early-challenge cohort (6-dc) or 20 days for the late-challenge birds $(20-\mathrm{dc})$. Birds challenged at 6 days (Figure 1C) showed incomplete colonization at $2 \mathrm{dpi}$ (2/7 and 3/7 for the control and GOS cohorts, respectively), but the treatment groups all showed complete colonization with C. jejuni at 9 dpi. Mean colonization levels of $6.4 \log _{10} \mathrm{CFU} / \mathrm{g}$ for the control diet and $5.9 \log _{10} \mathrm{CFU} / \mathrm{g}$ for the GOS diet were recorded (15 days). The birds then remained colonized thereafter to the end of the 35 days rearing period with no significant differences between the colonization levels of the dietary groups at any time (Figure 1C). The 20-dc birds (Figure 1D) were all found colonized at $8 \mathrm{dpi}$ with mean colonization levels of $6.8 \log _{10} \mathrm{CFU} / \mathrm{g}$ for the control and $7.2 \log _{10} \mathrm{CFU} / \mathrm{g}$ for the GOS diet (28 days). Viable counts of Campylobacter in cecal content remained high at the end of the 

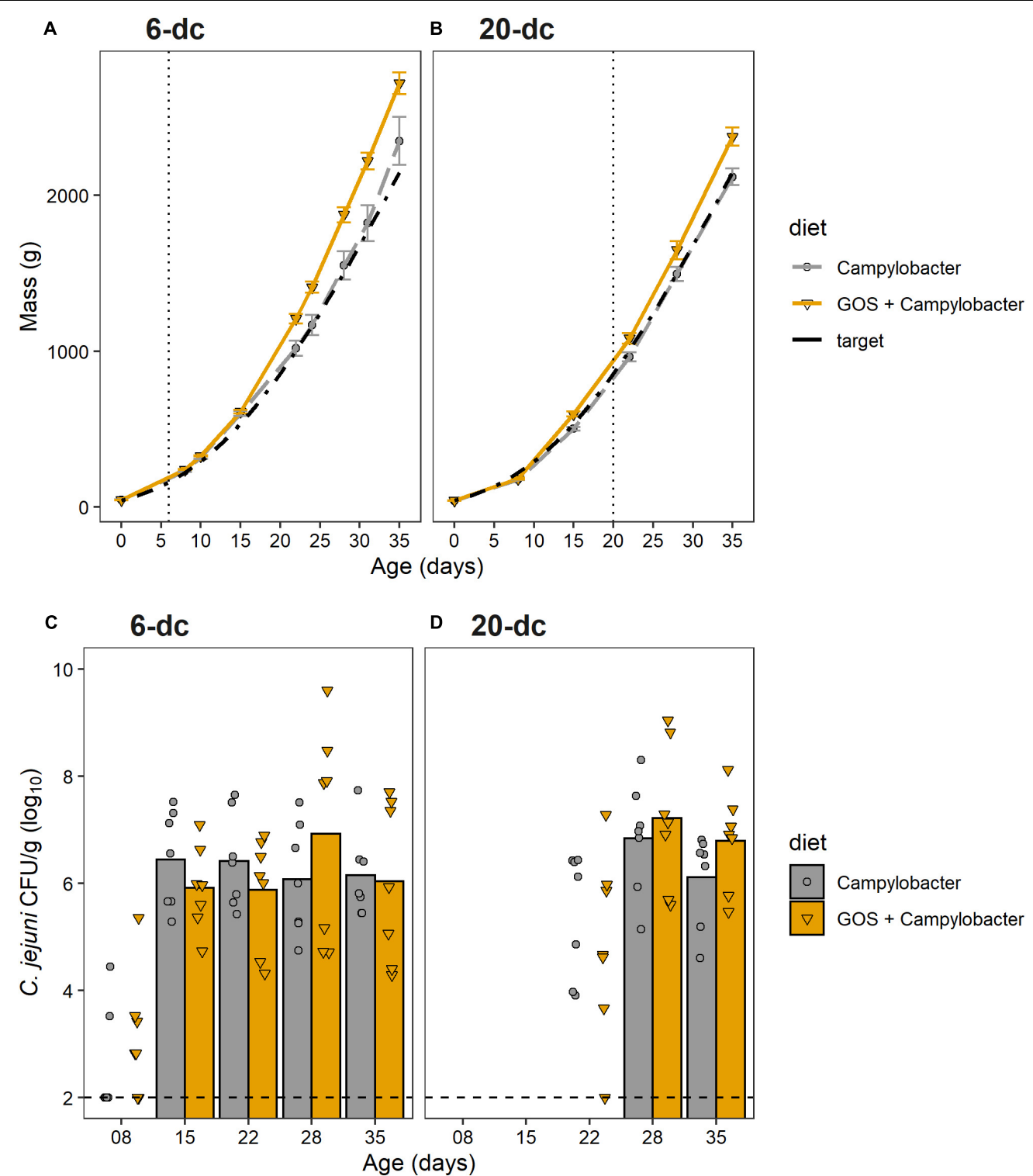

FIGURE 1 | GOS improves growth performance of broiler chickens, but does not affect $C$. jejuni colonization of the ceca. (A,B) Chicken live total mass from day of hatch to 35 days for 6 -dc birds (A) and 20-dc birds (B). The plots show the performances of the experimental treatments GOS + Campylobacter (GOS diet) Campylobacter (control diet) and the Ross 308 performance objective as the Target (Aviagen Performance Objectives, 2014). The dashed line indicates age at challenge. (C,D) Viable counts of Campylobacter recovered from the cecal content of 6-dc birds (C) and 20-dc birds (D). Data markers indicate Campylobacter CFU isolated from individual birds. Bars indicate mean Campylobacter CFU, excluding cohorts where Campylobacter levels were below the limit of detection. There are no significant differences in the Campylobacter counts for the GOS diet compared to control diet post colonization $(p>0.05)$. The dashed line indicates minimum level of detection.

rearing period with no significant differences between the diets at any time, independent of the age of challenge.

\section{Intestinal Villus and Crypt Metrics Were Affected by Dietary Galacto-Oligosaccharide Post-infection With Campylobacter jejuni}

Villus and crypt metrics were determined from 10 well-oriented villi for 3 to 4 birds from each group in a blind assessment of formalin-fixed H\&E-stained ileum sections. Measurement comparisons of the GOS and control diet groups for 6-dc birds showed greater villus length at $8(2 \mathrm{dpi} ; p=0.04)$ and 15 days ( $9 \mathrm{dpi} ; p=0.002$ ) for the C. jejuni colonized GOS-fed chickens compared to the $C$. jejuni colonized birds on control feed (Table 2). By 22 days the villus lengths of the 6-dc treatment groups were not significantly different and remained so until the end of the trial at 35 days. Comparison of the crypt depth measurements demonstrated that the GOS-fed birds at 15 days $(p=0.005)$ had significantly deeper crypts than the birds on the 


\begin{tabular}{|c|c|c|c|c|c|c|c|c|c|c|}
\hline & \multicolumn{10}{|c|}{ Histology measurements } \\
\hline & 8 days & SD & 15 days & SD & 22 days & SD & 28 days & SD & 35 days & SD \\
\hline \multicolumn{11}{|l|}{ Villus length $(\mu \mathrm{m})$} \\
\hline Campy (6-dc) & 550 & 74 & 560 & 64 & 803 & 33 & 940 & 71 & 934 & 32 \\
\hline Campy + GOS (6-dc) & 671 & 15 & 796 & 58 & 824 & 61 & 814 & 63 & 888 & 40 \\
\hline Campy (20-dc) & - & & - & & 636 & 56 & 746 & 88 & 954 & 98 \\
\hline Campy + GOS (20-dc) & - & & - & & 766 & 82 & 922 & 114 & 1092 & 124 \\
\hline$p$-value (6-dc) & 0.04 & & 0.002 & & 0.56 & & 0.09 & & 0.31 & \\
\hline$p$-value (20-dc) & - & & - & & 0.04 & & 0.05 & & 0.13 & \\
\hline \multicolumn{11}{|l|}{ Crypt depth $(\mu \mathrm{m})$} \\
\hline Campy (6-dc) & 117 & 12 & 111 & 7 & 125 & 8 & 128 & 5 & 121 & 8 \\
\hline Campy + GOS (6-dc) & 110 & 8 & 133 & 4 & 128 & 5 & 132 & 8 & 122 & 11 \\
\hline Campy (20-dc) & - & & - & & 82 & 16 & 92 & 24 & 104 & 22 \\
\hline Campy + GOS (20-dc) & - & & - & & 106 & 12 & 102 & 38 & 128 & 44 \\
\hline$p$-value $(6-\mathrm{dc})$ & 0.32 & & 0.005 & & 0.65 & & 0.49 & & 0.69 & \\
\hline$p$-value (20-dc) & - & & - & & 0.05 & & 0.67 & & 0.37 & \\
\hline \multicolumn{11}{|c|}{ Villus length/Crypt depth ratio (v/c) } \\
\hline Campy (6-dc) & 4.70 & 0.84 & 5.05 & 0.60 & 6.42 & 0.17 & 7.34 & 0.63 & 7.72 & 0.82 \\
\hline Campy + GOS (6-dc) & 6.10 & 0.38 & 5.98 & 0.39 & 6.44 & 0.31 & 6.17 & 0.59 & 7.28 & 0.27 \\
\hline Campy (20-dc) & - & - & - & - & 7.76 & 0.80 & 8.11 & 0.74 & 9.17 & 0.91 \\
\hline Campy + GOS (20-dc) & - & - & - & - & 7.23 & 0.91 & 9.04 & 0.82 & 8.53 & 0.72 \\
\hline$p$-value (6-dc) & 0.02 & & 0.04 & & 0.83 & & 0.08 & & 0.35 & \\
\hline$p$-value (20-dc) & - & & - & & 0.42 & & 0.14 & & 0.31 & \\
\hline
\end{tabular}

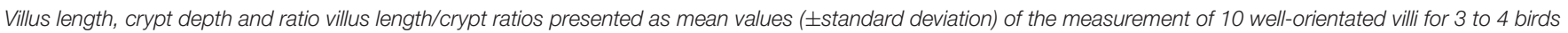
per group. Corresponding probabilities (p) were calculated using ANOVA tests, differences were considered significant at $p<0.05$.

control diet. Differences in the villus and crypt measurements affected differences in the villus to crypt ratios at 8 days $(p=0.02)$ and 15 days $(p=0.04)$ with the GOS-fed birds exhibiting greater ratios. For the 20-dc cohorts the GOS-fed birds also exhibited significant increases in villus height compared to the control diet after $C$. jejuni colonization at 22 days ( $2 \mathrm{dpi} ; p=0.04)$ and 28 days ( $8 \mathrm{dpi} ; p=0.05$ ). A significant increase in the crypt depth for the GOS-fed birds over the birds on the control diet was also recorded at 22 days $(p=0.05)$, but not thereafter. These differences did not result in significant differences in the villus to crypt ratios for the 20-dc experiment.

\section{Dietary Galacto-Oligosaccharide Modulates IL-17 Transcription Post Campylobacter Colonization}

Immuno-modulatory effects of dietary GOS on the innate immune responses of intestinal tissues from $C$. jejunicolonized broilers were assessed. RNAs were extracted from biopsies collected from ileal and cecal tissues to enable RT-PCR quantification of cytokine and chemokine gene transcripts representing the major inflammatory pathways of chickens. Cytokines IL-17A, IL-17F, IL-6, IL$1 \beta$ and chemokines ChCXCLi-1, ChCXCLi-2 (also known as ChIL-8) have previously been described as markers of the Th17 pathway (Reid et al., 2016). IFN- $\gamma$ is related to the Th1 pathway and the anti-inflammatory cytokine IL-10 is largely expressed from regulatory $\mathrm{T}$ cells (Treg) in chickens to control the inflammatory effects of the Th cell responses.

The innate immune response of ileal tissues for the early challenged birds $(6-\mathrm{dc})$ was characterized by modulation in the expression of IL-17A in C. jejuni-colonized birds on control feed compared to C. jejuni-colonized GOS-fed birds (Figure 2A). Following Campylobacter challenge IL-17A transcript levels were far greater in the GOS-fed birds (884-fold difference) than those on the control diet at 8 days ( $2 \mathrm{dpi}$; $p$-adj=0.005). By 15 days the difference had declined to 12 -fold due to an increase in IL$17 \mathrm{~A}$ in the C. jejuni-colonized birds on the control diet (9 dpi; $p$-adj $=0.02)$. The rise in IL-17A continued in the birds on the control diet until 35 days such that a significant difference was recorded in favor of the control diet birds from 22 days (16 dpi; $p$-adj =0.02), whilst the IL-17A levels were maintained in the GOS-fed birds throughout the time course. The proinflammatory cytokine IL-17F exhibited a significant increase in expression at 22 days $(16 \mathrm{dpi} ; p$-adj $=0.03)$ and 28 days (22 dpi; $p$-adj $=0.005$ ) in the control birds. Over the transition period, the regulatory cytokine IL-10 expression was significantly greater at 15 days (9 dpi; $p$-adj $=0.05$ ) and 22 days (16 dpi; $p$-adj $=0.03)$ in the C. jejuni-colonized birds on control diet compared to the GOS diet (GOS + Campylobacter treatment). For the late challenged birds (20-dc; Figure 2B), IL-6 was recorded as significantly greater for the $C$. jejuni-colonized birds on the control diet at 35 days ( $29 \mathrm{dpi}$; $p$-adj =0.005), largely owing to a fall in the IL-6 levels of the birds on the GOS diet (GOS + Campylobacter treatment). 

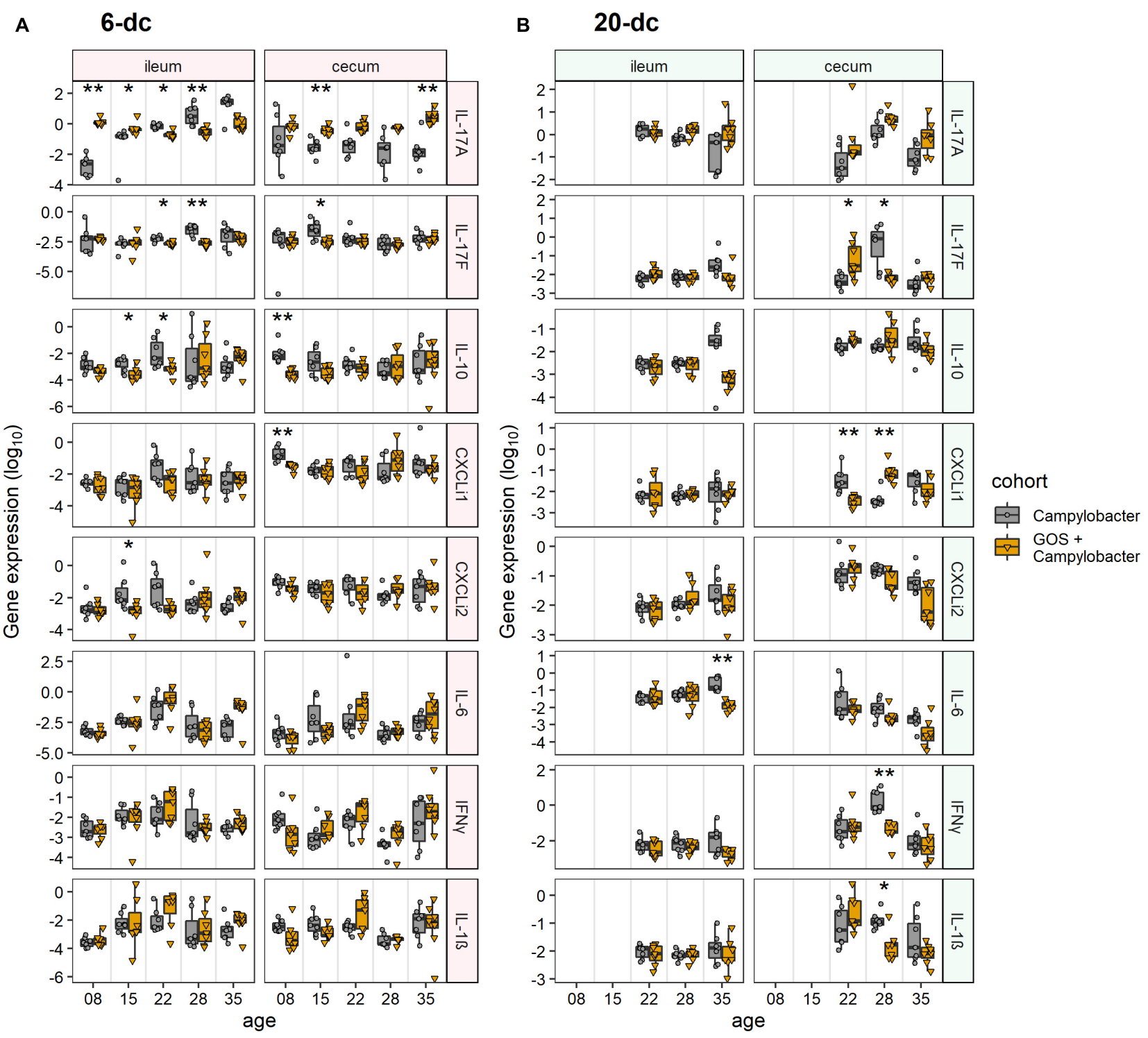

FIGURE 2 | Modulation of cecal and ileal innate immune responses to dietary GOS in Campylobacter-challenged broiler chickens. The figures report cytokine and chemokine normalized gene expression for 6-dc birds (A) and 20-dc birds (B) fed a control diet (Campylobacter treatment) or a GOS diet (GOS + Campylobacter treatment) recorded as $\log _{10}$ of the ratio for gene of interest/GAPDH from qPCR data of individual birds. Corresponding probabilities ( $p$-adj) were calculated using non-parametric Wilcoxon rank sum tests with Benjamini-Hochberg FDR correction, where differences at $p$-adj $<0.05$ were considered significant and are summarized in the plot using asterisks $\left({ }^{*} p\right.$-adj $\leq 0.05 ; * *$-adj $\left.\leq 0.01\right)$.

In cecal tissues IL-17A expression was also maintained in the GOS-fed birds post $C$. jejuni colonization. In the early challenge experiment (6-dc) IL-17A expression levels declined in the birds fed the control diet throughout the time course with significant differences recorded at 15 days $(9 \mathrm{dpi} ; p$-adj $=0.005)$ and 35 days (288-fold at $29 \mathrm{dpi}$; -adj $=0.01)$ compared to the birds on the GOS containing diet (Figure 2A). Pro-inflammatory IL-17F exhibited a significant increase at 15 days in the C. jejunicolonized birds fed the control diet compared to the birds on the GOS diet $(9 \mathrm{dpi} ; p$-adj $=0.04)$. This was preceded by differential increases at 8 days in IL-10 ( $2 \mathrm{dpi} ; p$-adj $=0.002)$ and ChCXCLi-1 $(2 \mathrm{dpi} ; p$-adj $=0.002)$. The late challenged birds $(20-\mathrm{dc})$ featured a significant switch in the expression of IL-17F from low to high for the $C$. jejuni-colonized birds on the control diet between $22 \mathrm{da}(2 \mathrm{dpi} ; p$-adj $=0.04)$ and 28 days $(8 \mathrm{dpi} ; p$-adj $=0.02)$, and conversely the birds on the GOS diet exhibited a high to low change in gene expression over the period (treatment GOS + Campylobacter). Increases in IL-17F were accompanied by significant increases in IFN- $\gamma(8 \mathrm{dpi} ; p-a d j=0.005)$ and IL$1 \beta$ (8 dpi; $p$-adj $=0.02)$ at $22 \mathrm{da}$ for the $C$. jejuni-colonized birds on the control diet. Transcription of the chemokine ChCXCLi1 was observed to exhibit the opposite trend to IL-17F over the 22 days $(2 \mathrm{dpi}$; $p$-adj $=0.005)$ to 28 days $(8 \mathrm{dpi}$; $p$ - $a d j=0.005)$ transition, with a fall in the mean expression value for the 
C. jejuni-colonized birds on the control diet compared to an increase in the $C$. jejuni-colonized GOS-fed birds (treatment GOS + Campylobacter in Figure 2B).

\section{Galacto-Oligosaccharide -Induced Microbiota Diversity Shifts in Campylobacter-Challenged Birds}

At 2 dpi ( 8 days) the $\alpha$-diversity of the cecal microbiota of 6-dc birds was lower in GOS + Campylobacter treated birds than the $C$. jejuni-colonized birds on the control diet (treatment Campylobacter), as indicated by lower Shannon entropy $(p=0.046)$ and inverse Simpson index $(p=0.022$; Figure $\mathbf{3 A}$ ). This may be attributed to the dietary GOS as the inverse Simpson's index of mock-challenged birds on a GOS diet was also lower than that of the Campylobacter treatment birds on the control diet $(p=0.032)$, with the difference observed in the corresponding Shannon entropy values approaching the significance threshold $(p=0.058)$. Shannon entropy was also lower in GOS + Campylobacter treatment birds at 28 days (22 dpi; $p=0.009)$ and 35 days ( $29 \mathrm{dpi} ; p=0.038)$ as presented in Figure 3A. There were no other observed differences in $\alpha$-diversity or species richness (Chao) in 6-dc birds. In the 20 dc experiment at 22 days ( $2 \mathrm{dpi}$ ) the inverse Simpson index of the cecal microbiota of the GOS + Campylobacter treatment birds was significantly greater than Campylobacter treatment birds $(p=0.0469$; Figure 3B). The responses recorded for the inverse Simpson index at $2 \mathrm{dpi}$ for the 6 -dc and 20-dc experiments show opposite trends, however, a similar trend can be noted in the higher $\alpha$-diversity of 6-dc GOS + Campylobacter treatment birds at the same age (22 days), although these changes did not reach significance. The modulation in $\alpha$-diversity at this age could be attributable to changes in host development or changes in diet formulation of grower to finisher related to husbandry.

Comparison of the phylum composition of 6-dc birds at 8 days $(2 \mathrm{dpi}$ ) indicates that the cecal microbiota of Campylobacter treatment birds was significantly different from the microbiota of the GOS + Campylobacter treatment birds $(p=0.0003)$ (Figure 3C). Comparison of the microbiota of GOS + Campylobacter treatment birds with the cecal microbiota of age-matched mock-challenged birds on the GOS diet alone at 8 days $(2 \mathrm{dpi})$ did not reveal any phyla-level differences ( $p=0.1613)$, whilst mock-challenged birds on either control or GOS diets also had different phyla compositions $(p=0.0096)$. These data suggest that the differences in microbiota ecology are linked to dietary GOS and not Campylobacter-colonization per se. No difference in phylum-composition was determined in 6 - $\mathrm{dc}$ birds at 15 days $(9 \mathrm{dpi} ; p=0.301), 22$ days ( $16 \mathrm{dpi}$; $p=0.69)$ or 35 days $(p=0.055)$. However, the composition of the cecal microbiota of birds from the GOS + Campylobacter and the Campylobacter treatment groups were different at 28 days (22 dpi; $p=0.0004$ ), which likely corresponds with the reverse in $\alpha$-diversity first observed at this age. No differences were determined in the phylum-composition of 20 -dc birds at $2 \mathrm{dpi}$ $(p=0.742)$ (Figure 3D).

At OTU level 16S rRNA gene sequencing reads were clustered at $97 \%$ similarity, which serves as a proxy for species-level distinction. The 6 -dc birds at 8 days ( 2 dpi) show a key differential OTU between Campylobacter-colonized birds on the control diet (Campylobacter treatment) versus C. jejuni-colonized birds on the GOS diet (GOS + Campylobacter treatment) had strong sequence similarity to Escherichia coli (OTU0001; 99.21\%, Supplementary Figure S2A). OTU0001 did not discriminate the microbiota of the Campylobacter-challenged birds on the control diet (Campylobacter treatment) from the microbiota of mockchallenged birds on the GOS diet (Supplementary Figure S2C). In addition, previous analysis of the mock-challenged controls by our laboratory indicated that Lactobacillus johnsonii outcompetes L. crispatus in GOS-fed birds (Richards et al., 2019b). In the analysis presented here OTU0002 has strong sequence similarity with the L. crispatus strain (97.21\%) and OTU0017 has a strong sequence similarity with $L$. johnsoniii (98.03\%). In 6-dc birds at 8 days $(2 \mathrm{dpi}$ ) both OTU0002 (L. crispatus) and OTU0017 (L. johnsoniii) are associated with dietary GOS (Supplementary Figure S2B). However, L. johnsoniii (OTU0017) continues to be associated with dietary GOS at: 15 days ( 9 dpi), 22 days (16 dpi) and 28 days (22 dpi). Whereas, L. crispatus (OTU0002) is later associated with the control diet at 15 days $(9 \mathrm{dpi})$ and 35 days (29 dpi).

\section{DISCUSSION}

Concerns are growing regarding the over use of antimicrobials in animal production, and any concomitant increase in risk of antimicrobial resistance transferring to humans (Landers et al., 2012). These concerns have brought about calls for restricting antibiotic use in food producing animals (Tang et al., 2017). Although the European Union banned antimicrobial growth promoters since 2006, the practice continues in many countries and has prompted calls for a worldwide ban, particularly in the poultry and pig industries. It is becoming increasingly evident that improved on farmperformance at the expense of intestinal health and the zoonotic dissemination of antimicrobial resistance cannot continue (Awad et al., 2015, 2017; Smith et al., 2016). Under increasing economic pressure, the poultry industry is pursuing effective alternative methods to promote bird growth whilst controlling farm sources of antimicrobial resistance and zoonotic disease. Prebiotic feed additives such as GOS, a byproduct of the dairy industry, have revealed potential growthpromoting effects in piglets (Alizadeh et al., 2016) and chickens (Richards et al., 2019b), whilst fructo-ologosaccharide (FOS) have been reported to reduce Salmonella colonization of chicks (Fukata et al., 1999). Here, we assess the impact of a GOS prebiotic on chickens colonized by $C$. jejuni at either 6 or 20 days with respect to their zootechnical performance, gut architecture and differences in intestinal cytokine and chemokine transcription (the experimental designs and corresponding data are summarized in Figure 4).

Two independent trials show significant differences in live bodyweights of $C$. jejuni-challenged birds, the birds fed GOS additive were significantly heavier at the typical market age of 35 days compared to a calorie-match control diet. GOS 

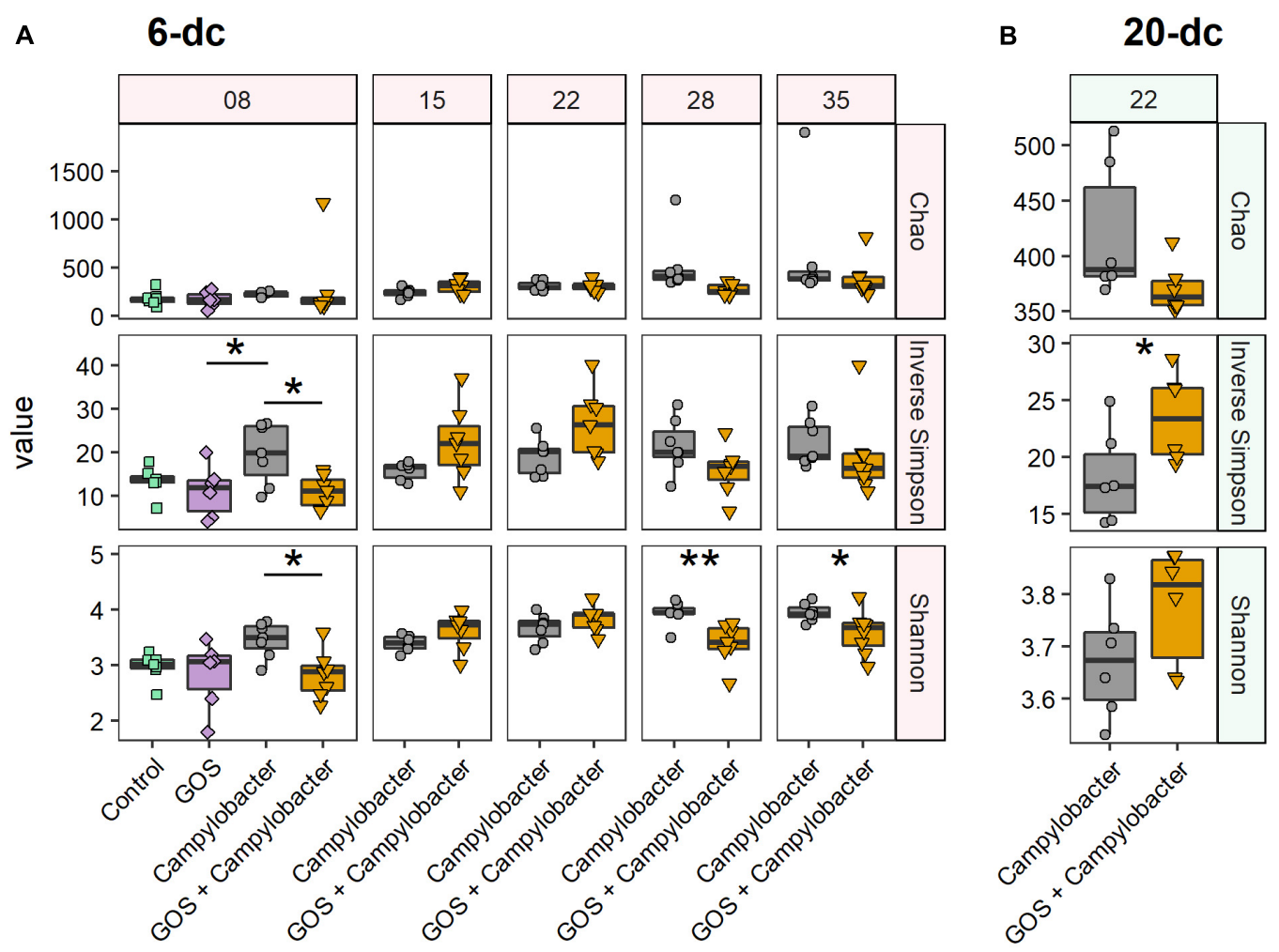

C

6-dc

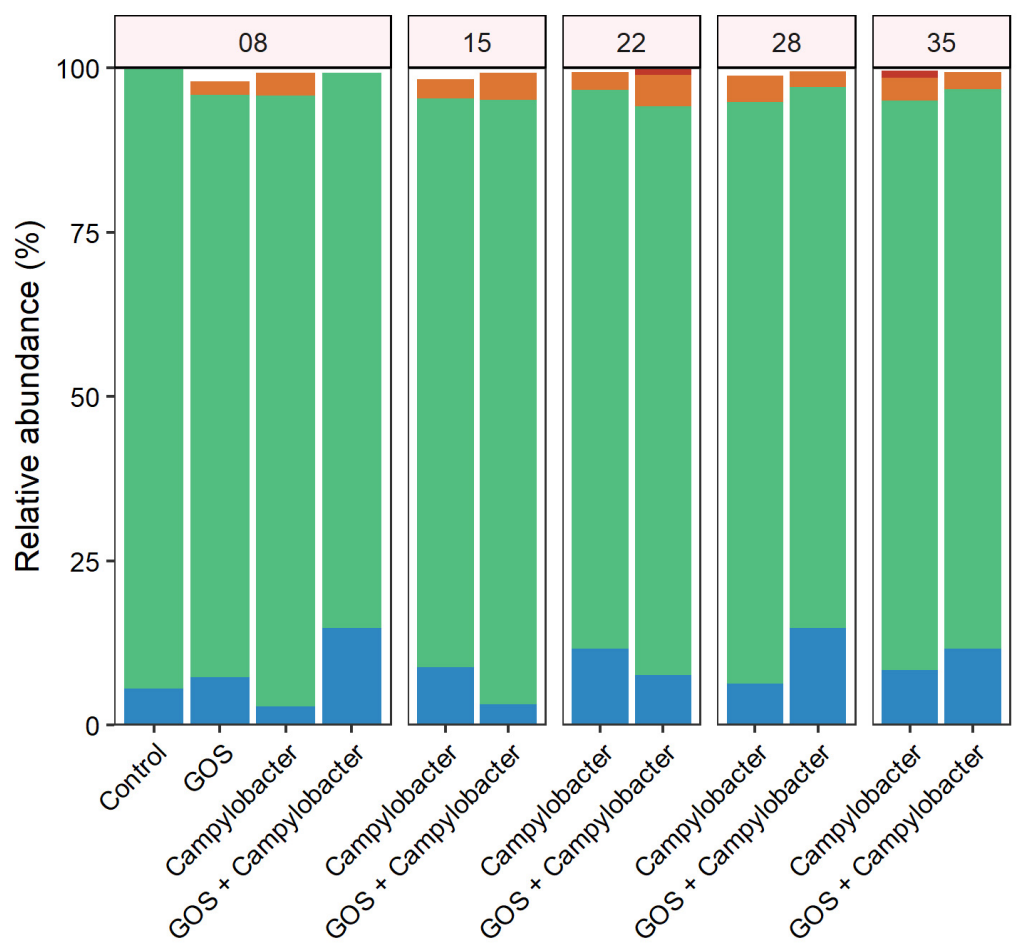

D

20-dc

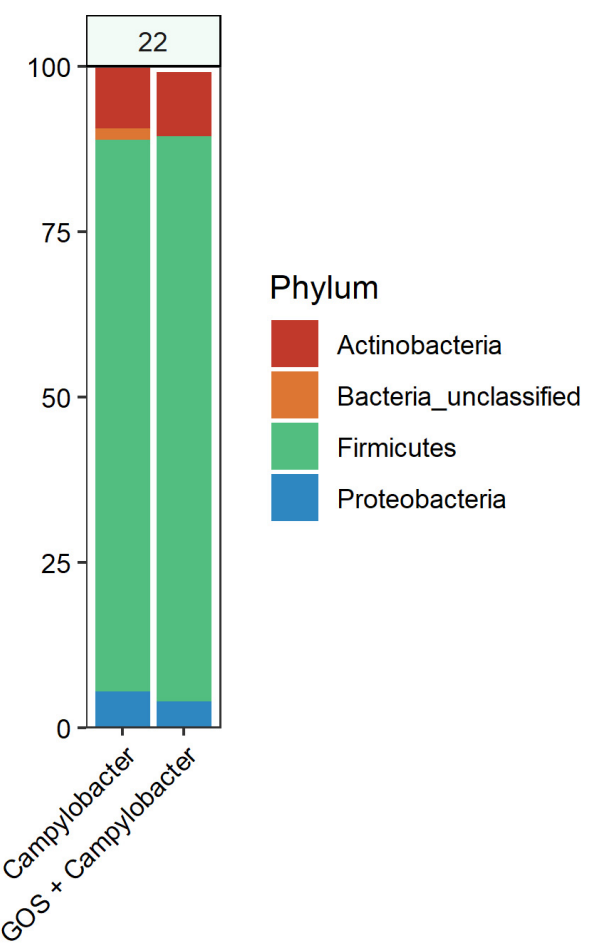

FIGURE 3 | Dietary GOS promotes differential shifts in the cecal microbiota post Campylobacter challenge. (A,B) Cecal $\alpha$-diversity for 6 -dc birds (A) and 20-dc birds (B) described as Chao richness, Inverse Simpson diversity and Shannon diversity (as indicated by panels on right hand side of the figure). The age of the birds are in days as indicated by the numerals in the strip at the top of the figure. Significant differences between groups are indicated by asterisks $\left({ }^{*} p \leq 0.05\right.$; $\left.{ }^{* *} p \leq 0.01\right)$. (C,D) OTU relative abundance for 6 -dc birds (C) and 20-dc birds (D) summarized at Phyla level. 


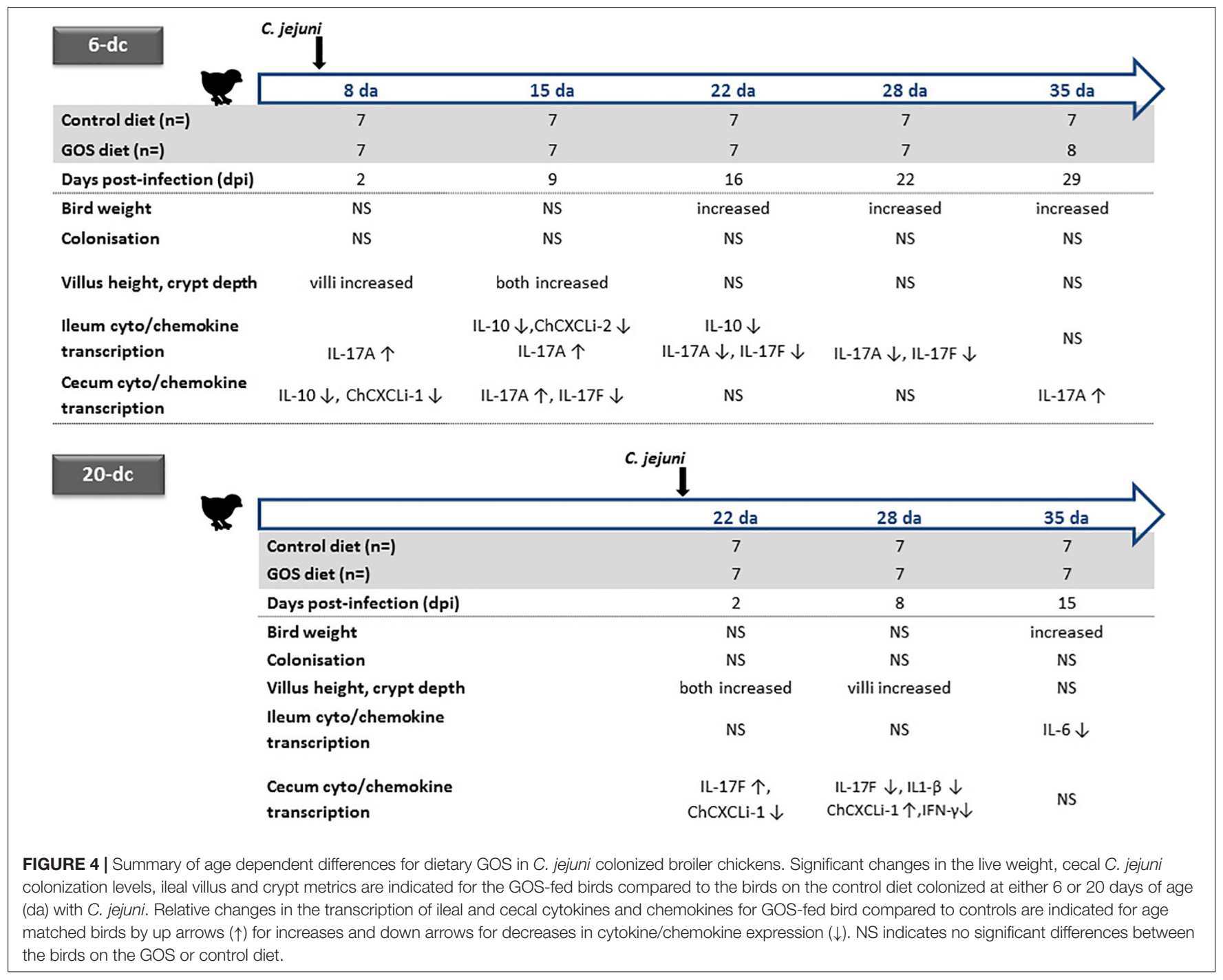

supplementation improved the growth rate performance of Campylobacter-colonized broiler chickens irrespective of the timing of the challenge with C. jejuni at either 6 days or 20 days of age. However, dietary GOS inclusion did not prevent or reduce C. jejuni HPC5 colonization of broiler chickens GIT within the 35 days lifespan of the birds. C. jejuni HPC5 is a broiler chicken isolate that has been used routinely and reliably to colonize the intestinal tract of broiler chickens (Loc Carrillo et al., 2005; Scott et al., 2007; Connerton et al., 2018). In recent years several studies have employed 16S rRNA gene sequences to comprehensively document changes in the cecal microbiota that accompany Campylobacter-colonization of broilers. These studies have noted differences in the relative abundance of Bifidobacterium, Lactobacillaceae, Clostridium cluster XIVa and Mollicutes, with transient age related shifts in specific members of the Lachnospiraceae and Ruminococcaceae (Thibodeau et al., 2015, 2017; Connerton et al., 2018; Richards et al., 2019a). As an extension of the outputs from these studies it was recognized that transitions in the cecal microbiota were evident between
14 and 18 days that coincide with the reduced availability of maternal antibodies, and represent a window of opportunity for the entry for bacteria to bloom and new intestinal microbes to become established that can affect changes in gut health (Awad et al., 2016; Connerton et al., 2018; Ijaz et al., 2018). Early prebiotic diets offer the prospect of achieving a stable microbiota that can resist opportunist expansion or colonists. Prebiotic oligosaccharides have previously been reported to reduce the cecal $C$. jejuni colonization loads of broiler chickens, for example the use of a chicory fructan additive for 42 days in male Ross 308 birds (Yusrizal and Chen, 2003), or as the use of mannan-oligosaccharide (MOS) with male Cobb 500 birds at 34 days (Baurhoo et al., 2009). It is conceivable that improved broiler breeding programs and optimized diets will produce heavier birds faster, which will require fast-resolving methods that reduce or displace unwelcome gut bacteria.

Intestinal histomorphometric parameters are considered indicators of gut health whereby a healthy ileal mucosa should display long villi with high villus/crypt ratios. A transient increase 
in villus length and crypt depth was observed over the first 2 weeks post-challenge for birds sustained on the GOS diet irrespective of the timing of Campylobacter challenge. Increases in the absorption surface provide a favorable environment for nutrient uptake leading to efficient feed utilization. Several studies have shown the beneficial effects of dietary additives on Ross 308 broiler chicken villus architecture and body weight gain during heat stress challenges such as prebiotic supplements (Silva et al., 2010), alpha-lipoic acid additive (Yoo et al., 2016), or probiotic mixtures (Song et al., 2014). Similarly, the data presented here suggests that nutrient absorption competence associated with the development of villi in the small intestine of birds fed the GOS diet, leads to an increase in body weight despite gut colonization by C. jejuni.

Intestinal mucosa constitutes a physical and immunological protective barrier for the integrity of the intestinal tract to prevent infection by pathogens and maintain an environment that can sustain a healthy and productive microbiota. However, the composition of the gut microbiota is under surveillance of the mucosal innate and adaptive immune systems (Kraehenbuhl and Neutra, 1992). Numerous immune cell populations such as regulatory T-cells (Treg), Th17 cells, IgA-secreting plasma cells, natural killer cells (NK), macrophages, dentritic cells (DCs), innate lymphoid cells (ILCs) contribute to host defense against infection with pathogenic microbes (Honda and Littman, 2012). Members of the IL-17 family of cytokines, IL-17A and IL-17F are produced by a subset of CD4 $+\mathrm{T}$ cells named Th17, and have more recently been associated in the gut with dendritic cells and group 3 innate lymphoid cells (ILC3) (Li S. et al., 2018). While they beneficially mediate resistance to extracellular bacterial and fungal infection via enhanced mucosal production of mucus and antimicrobial peptides, IL-17A and IL-17F are also involved in several autoimmune disorders (Bettelli et al., 2007; Yang et al., 2008; Ishigame et al., 2009).

Here, we demonstrate dietary GOS inclusion can maintain transcript levels of IL-17A and suppress IL-17F post colonization with C. jejuni. Previously we have shown C. jejuni challenge at 6 days triggered a transient increases in IL-17A and IL-17F in broiler chickens at 15 days ( $9 \mathrm{dpi}$ ) compared with non-colonized birds (Connerton et al., 2018), and more recently that dietary GOS increases the expression of IL-17A in juvenile birds up to 15 days (Richards et al., 2019b). In mice IL-17A expression has been proposed to benefit intestinal barrier function and IL-17F to weaken intestinal integrity, since IL-17A inhibition exacerbates induced colitis (Maxwell et al., 2015) and IL-17F suppression is protective (Tang et al., 2018). Evidence suggests the modulation of IL-17A is associated with the regulation of the tight junction formation and regulation of the mucosal barrier via activation of the ERK MAPK pathway (Awane et al., 1999; Cario et al., 2000; Kinugasa et al., 2000). C. jejuni colonization of the chicken intestine appears to result in the expression of both the IL-17 subtypes with opposing effects mediated at different times. The dominant response may in part explain why there are differences in the impact of C. jejuni colonization reported for different bacterial types on various broiler chicken breeds (Humphrey et al., 2014, 2015). An increase of IL-17A in response to dietary GOS in the presence or absence of C. jejuni colonization may well benefit intestinal health and contribute to the increased growth rate observed for GOS-fed birds. GOS does not prevent $C$. jejuni colonization but may also lead to performance improvements by suppression of IL-17F under circumstances when $C$. jejuni colonization has been reported to be associated with bird health and productivity (Bull et al., 2008). These current studies were conducted in clean controlled biosecure facilities such that the birds would not be subject to exposure to endemic viral, bacterial and protozoal pathogens that are frequently encountered by commercial broiler chickens. Under these circumstances the impact of prebiotic priming of intestinal innate immunity may be of greater importance in field applications.

Consistent with previous reports we observed GOS-driven changes in the cecal microbiota of chickens featuring specific operational taxonomic units identifiable as lactobacilli (Hughes et al., 2017; Azcarate-Peril et al., 2018). Colonization by C. jejuni did not prevent the differential increase in abundance of Otu0017 (L. johnsoniii) associated previously with dietary GOS (Richards et al., 2019b). At 2dpi for the 6-dc birds Otu0002 (L. crispatus) and Otu0017 (L. johnsoniii) showed increases in abundance in association with the GOS-diet. However, Otu0017 (L. johnsoniii) remained an abundant member of the GOS + Campylobacter treatment group over the course of the experiment, and in contrast the relative abundance of Otu0002 (L. crispatus) fell such that it exhibited significantly greater abundance at 9 and 29 dpi in the Campylobacter treatment group fed the control diet. These data are consistent with L. johnsonii outcompeting L. crispatus in GOS-fed birds, and consistent with the hypothesis that L. johnsonii contributes to the stability of the innate immune response observed in GOSfed birds. L. johnsonii is an established probiotic species that has been reported to improve growth performance, intestinal development, and act as competitive exclusion agent against bacterial pathogens in broiler chickens (La Ragione et al., 2004; Wang et al., 2017).

IL-17A responses to probiotics have been reported for ex vivo Peyer's patch stimulated $\mathrm{T}$ cells derived from mice orally administered with lactic acid bacteria ( $L$. bulgaricus or Streptococcus thermophilus). Over 7 days the stimulated T cells exhibited increases in the levels of IL-17 whilst IL-10 and Th2 IL-4 remained unchanged (Kamiya et al., 2016). In chickens transient IL-17 induction has been observed during the natural development the intestinal microbiota (Crhanova et al., 2011). In the absence of IL-22, pro-inflammatory Th17 induction did not result in intestinal damage but upon Salmonella Enteritidis challenge tissue damage was observed as a result of a Th17 response that features the cytokines IL17 and IL-22.

\section{CONCLUSION}

In conclusion the data support the contention that GOS dietinduced microbiota shifts can: (1) Improve the growth rate 
of broiler chickens independent of C. jejuni colonization. (2) Maintain ileal and cecal IL-17A transcription that can positively influence gut health in the presence of C. jejuni. (3) Suppress IL-17F expression arising as a consequence $C$. jejuni colonization that has the potential to impair gut integrity and health.

\section{DATA AVAILABILITY STATEMENT}

All 16S rDNA sequence data originally reported here is available at under accessions SRR10059315 to SRR10059356 in NCBI SRA study SRP133552. All other $16 \mathrm{~S}$ rDNA sequences reported in this study are also available from NCBI SRA study SRP133552. Raw zootechnical and qPCR gene expression data is available from https://github.com/PJRichards/lafontaine_campy_gos.

\section{ETHICS STATEMENT}

Experiments involving the use of birds were subjected to approval process under National Guidelines by the United Kingdom Home Office. Work on this project was approved under United Kingdom Government Home Office Project Licensing ASPA 86. The project license has been reviewed and approved by the University Ethics Committee prior to submission to the Home Office, which includes the scrutiny of animal welfare, ethics and handling.

\section{AUTHOR CONTRIBUTIONS}

NF contributed to the study design. NF and IC conceived and designed the experiments. GF, PR, PC, PO'K, NG, NC, and IC performed the experiments. GF, PR, PC, and IC analyzed the

\section{REFERENCES}

Alizadeh, A., Akbari, P., Difilippo, E., Schols, H. A., Ulfman, L. H., Schoterman, M. H. C., et al. (2016). The piglet as a model for studying dietary components in infant diets: effects of galacto-oligosaccharides on intestinal functions. Br. J. Nutr. 115, 605-618. doi: 10.1017/S0007114515004997

Andreoletti, O., Budka, H., Buncic, S., Collins, J. D., Griffin, J., Hald, T., et al. (2010). Scientific opinion on quantification of the risk posed by broiler meat to human campylobacteriosis in the EU. EFSA J. 8:1437. doi: 10.2903/j.efsa.2010.1437

Aviagen Performance Objectives (2014). Ross 308 Broiler Performance Objectives. Scotland, UK: Aviagen Limited Newbridge, Midlothian EH28 8SZ.

Awad, W. A., Hess, C., and Hess, M. (2017). Enteric pathogens and their toxininduced disruption of the intestinal barrier through alteration of tight junctions in chickens. Toxins 9:E60. doi: 10.3390/toxins 9020060

Awad, W. A., Mann, E., Dzieciol, M., Hess, C., Schmitz-Esser, S., Wagner, M. et al. (2016). Age-related differences in the luminal and mucosa-associated gut microbiome of broiler chickens and shifts associated with Campylobacter jejuni infection. Front. Cell. Infect. Microbiol. 6:154. doi: 10.3389/fcimb.2016. 00154

Awad, W. A., Smorodchenko, A., Hess, C., Aschenbach, J. R., Molnár, A., Dublecz, K., et al. (2015). Increased intracellular calcium level and impaired nutrient absorption are important pathogenicity traits in the chicken intestinal epithelium during Campylobacter jejuni colonization. Appl. Microbiol. Biotechnol. 99, 6431-6441. doi: 10.1007/s00253-015-6543-z

Awane, M., Andres, P. G., Li, D. J., and Reinecker, H. C. (1999). NF-кBinducing kinase is a common mediator of IL- 17-, TNF- $\alpha$-, and IL- $1 \beta$-induced data and wrote the manuscript. All authors approved the final manuscript for publication.

\section{FUNDING}

The authors acknowledge the support from Saputo Dairy UK.

\section{ACKNOWLEDGMENTS}

The authors thank Darren L. Smith at University of Northumbria, Newcastle upon Tyne, for NGS sequencing.

\section{SUPPLEMENTARY MATERIAL}

The Supplementary Material for this article can be found online at: https://www.frontiersin.org/articles/10.3389/fmicb. 2019.03030/full\#supplementary-material

FIGURE S1 | Rarefaction curves indicating sampling efficiency of cecal bacterial communities. Collectors curved were constructed for $16 \mathrm{~S}$ rDNA sequences curated using mothur pipeline. Communities are presented by cohort as indicated by text in strip at the top of each panel. The colors indicate the rarefaction curves for individual bird in each cohort (key inset).

FIGURE S2 | GOS responsive OTUs in age-matched broilers. Discriminative OTUs were identified using LEfSE between 6-dc challenged birds: Treatments Campylobacter and GOS + Campylobacter (A), 20-dc challenged birds: Treatments Campylobacter and GOS + Campylobacter (B), and 6-dc Campylobacter-challenged and GOS mock-challenged control birds: Treatments Campylobacter and GOS (C). Comparisons of OTU relative abundance were made between age-matched cohorts that were rarefied to include only OTU $\geq 10$ reads. For clarity only OTU with $p<0.05$ for the embedded Kruskal Wallis ANOVA test and LDA $\left(\log _{10}\right)>2$ are reported (Mothur defaults).

chemokine promoter activation in intestinal epithelial cells. J. Immunol. 162, 5337-5344.

Azcarate-Peril, M. A., Butz, N., Cadenas, M. B., Koci, M., Ballou, A., Mendoza, M., et al. (2018). An attenuated Salmonella enterica serovar Typhimurium strain and galactooligosaccharides accelerate clearance of Salmonella infections in poultry through modifications to the gut microbiome. Appl. Environ. Microbiol. 84, 2526-2543. doi: 10.1128/AEM. 02526-17

Baurhoo, B., Ferket, P. R., and Zhao, X. (2009). Effects of diets containing different concentrations of mannanoligosaccharide or antibiotics on growth performance, intestinal development, cecal and litter microbial populations, and carcass parameters of broilers. Poult. Sci. 88, 2262-2272. doi: 10.3382/ps. 2008-00562

Bettelli, E., Oukka, M., and Kuchroo, V. K. (2007). TH-17 cells in the circle of immunity and autoimmunity. Nat. Immunol. 8, 345-350. doi: 10.1038/ni0407345

Broom, L. J., and Kogut, M. H. (2018). Inflammation: friend or foe for animal production? Poult. Sci. 97, 510-514. doi: 10.3382/ps/pex314

Bull, S. A., Thomas, A., Humphrey, T., Ellis-Iversen, J., Cook, A. J., Lovell, R., et al. (2008). Flock health indicators and Campylobacter spp. in commercial housed broilers reared in Great Britain. Appl. Environ. Microbiol. 74, 5408-5413. doi: 10.1128/AEM.00462-08

Caporaso, J. G., Lauber, C. L., Walters, W. A., Berg-Lyons, D., Lozupone, C. A., Turnbaugh, P. J., et al. (2011). Global patterns of $16 \mathrm{~S}$ rRNA diversity at a depth of millions of sequences per sample. Proc. Natl. Acad. Sci. U.S.A. 108, 4516-4522. doi: 10.1073/pnas.1000080107 
Cario, E., Rosenberg, I. M., Brandwein, S. L., Beck, P. L., Reinecker, H. C., and Podolsky, D. K. (2000). Lipopolysaccharide activates distinct signaling pathways in intestinal epithelial cell lines expressing Toll-like receptors. J. Immunol. 164, 966-972. doi: 10.4049/jimmunol.164.2.966

Clavijo, V., and Flórez, M. J. V. (2018). The gastrointestinal microbiome and its association with the control of pathogens in broiler chicken production: a review. Poult. Sci. 97, 1006-1021. doi: 10.3382/ps/pex359

Connerton, P. L., Richards, P. J., Lafontaine, G. M., O'Kane, P. M., Ghaffar, N., Cummings, N. J., et al. (2018). The effect of the timing of exposure to Campylobacter jejuni on the gut microbiome and inflammatory responses of broiler chickens. Microbiome 6:88. doi: 10.1186/s40168-0180477-5

Crhanova, M., Hradecka, H., Faldynova, M., Matulova, M., Havlickova, H., Sisak, F., et al. (2011). Immune response of chicken gut to natural colonization by gut microflora and to Salmonella enterica serovar enteritidis infection. Infect. Immun. 79, 2755-2763. doi: 10.1128/IAI.01375-10

EFSA (2018). The European Union summary report on trends and sources of zoonoses, zoonotic agents and food-borne outbreaks in 2017. EFSA J. 16:5500. doi: $10.2903 /$ j.efsa.2018.5500

Fukata, T., Sasai, K., Miyamoto, T., and Baba, E. (1999). Inhibitory effects of competitive exclusion and fructooligosaccharide, singly and in combination, on Salmonella colonization of chicks. J. Food Prot. 62, 229-233. doi: 10.4315/0362028x-62.3.229

Gormley, F. J., Bailey, R. A., Watson, K. A., McAdam, J., Avendaño, S., Stanley, W. A., et al. (2014). Campylobacter colonization and proliferation in the broiler chicken upon natural field challenge is not affected by the bird growth rate or breed. Appl. Environ. Microbiol. 80, 6733-6738. doi: 10.1128/AEM.02162-14

Honda, K., and Littman, D. R. (2012). The microbiome in infectious disease and inflammation. Annu. Rev. Immunol. 30, 759-795. doi: 10.1146/annurevimmunol-020711-074937

Hughes, R. A., Ali, R. A., Mendoza, M. A., Hassan, H. M., and Koci, M. D. (2017). Impact of dietary galacto-oligosaccharide (GOS) on chicken's gut microbiota, mucosal gene expression, and Salmonella colonization. Front. Vet. Sci. 4:192. doi: $10.3389 /$ fvets.2017.00192

Humphrey, S., Chaloner, G., Kemmett, K., Davidson, N., Williams, N., Kipar, A., et al. (2014). Campylobacter jejuni is not merely a commensal in commercial broiler chickens and affects bird welfare. mBio 5:e01364-14. doi: 10.1128/mBio. 01364-14

Humphrey, S., Lacharme-Lora, L., Chaloner, G., Gibbs, K., Humphrey, T., Williams, N., et al. (2015). Heterogeneity in the infection biology of Campylobacter jejuni isolates in three infection models reveals an invasive and virulent phenotype in a ST21 isolate from poultry. PLoS One 10:e0141182. doi: 10.1371/journal.pone.0141182

Ijaz, U. Z., Sivaloganathan, L., McKenna, A., Richmond, A., Kelly, C., Linton, M., et al. (2018). Comprehensive longitudinal microbiome analysis of the chicken cecum reveals a shift from competitive to environmental drivers and a window of opportunity for Campylobacter. Front. Microbiol. 9:2452. doi: 10.3389/fmicb. 2018.02452

Ishigame, H., Kakuta, S., Nagai, T., Kadoki, M., Nambu, A., Komiyama, Y., et al. (2009). Differential roles of interleukin-17A and $-17 \mathrm{~F}$ in host defense against mucoepithelial bacterial infection and allergic responses. Immunity 30 , 108-119. doi: 10.1016/j.immuni.2008.11.009

Jung, S. J., Houde, R., Baurhoo, B., Zhao, X., and Lee, B. H. (2008). Effects of galacto-oligosaccharides and a Bifidobacteria lactis-based probiotic strain on the growth performance and fecal microflora of broiler chickens. Poult. Sci. 87, 1694-1699. doi: 10.3382/ps.2007-00489

Kaakoush, N. O., Castaño-Rodríguez, N., Mitchell, H. M., and Man, S. M. (2015). Global epidemiology of Campylobacter infection. Clin. Microbiol. Rev. 28, 687-720. doi: 10.1128/CMR.00006-15

Kaiser, P., Underwood, G., and Davison, F. (2003). Differential cytokine responses following Marek's disease virus infection of chickens differing in resistance to Marek's disease. J. Virol. 77, 762-768. doi: 10.1128/jvi.77.1.762-768.2003

Kamiya, T., Watanabe, Y., Makino, S., Kano, H., and Tsuji, N. (2016). Improvement of intestinal immune cell function by lactic acid bacteria for dairy products. Microorganisms 5:E1. doi: 10.3390/microorganisms5010001

Kinugasa, T., Sakaguchi, T., Gu, X., and Reinecker, H. (2000). Claudins regulate the intestinal barrier in response to immune mediators. Gastroenterology 118, 1001-1011. doi: 10.1016/S0016-5085(00)70351-9
Kogut, M. H. (2009). Impact of nutrition on the innate immune response to infection in poultry. J. Appl. Poult. Res. 18, 111-124. doi: 10.3382/japr.200800081

Kogut, M. H., Genovese, K. J., Swaggerty, C. L., He, H., and Broom, L. (2018). Inflammatory phenotypes in the intestine of poultry: not all inflammation is created equal. Poult. Sci. 97, 2339-2346. doi: 10.3382/ps/pey087

Kozich, J. J., Westcott, S. L., Baxter, N. T., Highlander, S. K., and Schloss, P. D. (2013). Development of a dual-index sequencing strategy and curation pipeline for analyzing amplicon sequence data on the MiSeq Illumina sequencing platform. Appl. Environ. Microbiol. 79, 5112-5120. doi: 10.1128/AEM.01043-13

Kraehenbuhl, J.-P., and Neutra, M. R. (1992). Molecular and cellular basis of immune protection of mucosal surfaces. Physiol. Rev. 72, 853-879. doi: 10.1152/ physrev.1992.72.4.853

La Ragione, R. M., Narbad, A., Gasson, M. J., and Woodward, M. J. (2004). In vivo characterization of Lactobacillus johnsonii FI9785 for use as a defined competitive exclusion agent against bacterial pathogens in poultry. Lett. Appl. Microbiol. 38, 197-205. doi: 10.1111/j.1472-765X.2004.01474.x

Landers, T. F., Cohen, B., Wittum, T. E., and Larson, E. L. (2012). A review of antibiotic use in food animals: perspective, policy, and potential. Public Health Rep. 127, 4-22. doi: 10.1177/003335491212700103

Li, L., Pielsticker, C., Han, Z., Kubasová, T., Rychlik, I., Kaspers, B., et al. (2018). Infectious bursal disease virus inoculation infection modifies Campylobacter jejuni-host interaction in broilers. Gut Pathog. 10:13. doi: 10.1186/s13099-0180241-1

Li, S., Bostick, J. W., and Zhou, L. (2018). Regulation of innate lymphoid cells by aryl hydrocarbon receptor. Front. Immunol. 8:1909. doi: 10.3389/fimmu.2017. 01909

Livak, K. J., and Schmittgen, T. D. (2001). Analysis of relative gene expression data using real-time quantitative PCR and the 2- $\Delta \Delta \mathrm{CT}$ method. Methods 25 , 402-408. doi: 10.1006/meth.2001.1262

Loc Carrillo, C., Atterbury, R. J., El-Shibiny, A., Connerton, P. L., Dillon, E., Scott, A., et al. (2005). Bacteriophage therapy to reduce Campylobacter jejuni colonization of broiler chickens. Appl. Environ. Microbiol. 71, 6554-6563. doi: 10.1128/AEM.71.11.6554-6563.2005

Maxwell, J. R., Zhang, Y., Brown, W. A., Smith, C. L., Byrne, F. R., Fiorino, M., et al. (2015). Differential roles for interleukin-23 and interleukin-17 in intestinal immunoregulation. Immunity 43, 739-750. doi: 10.1016/j.immuni.2015.08.019

Miles, A. A., Misra, S. S., and Irwin, J. O. (1938). The estimation of the bactericidal power of the blood. J. Hyg. 38, 732-749. doi: 10.1017/S002217240001158X

Nang, N., Lee, J., Song, B., Kang, Y., Kim, H., and Seo, S. (2011). Induction of inflammatory cytokines and toll-like receptors in chickens infected with avian H9N2 influenza virus. Vet. Res. 42:64. doi: 10.1186/1297-9716-42-64

Newell, D. G., Elvers, K. T., Dopfer, D., Hansson, I., Jones, P., James, S., et al. (2011). Biosecurity-based interventions and strategies to reduce Campylobacter spp. on poultry farms. Appl. Environ. Microbiol. 77, 8605-8614. doi: 10.1128/AEM. 01090- 10

Osimani, A., Aquilanti, L., Pasquini, M., and Clementi, F. (2017). Prevalence and risk factors for thermotolerant species of Campylobacter in poultry meat at retail in Europe. Poult. Sci. 96, 3382-3391. doi: 10.3382/ps/pex143

Pedroso, A. A., Hurley-Bacon, A. L., Zedek, A. S., Kwan, T. W., Jordan, A. P. O., Avellaneda, G., et al. (2013). Can probiotics improve the environmental microbiome and resistome of commercial poultry production? Int. J. Environ. Res. Public Health 10, 4534-4559. doi: 10.3390/ijerph10104534

Pourabedin, M., and Zhao, X. (2015). Prebiotics and gut microbiota in chickens. FEMS Microbiol. Lett. 362:fnv122. doi: 10.1093/femsle/fnv122

R Core Team (2019). R: a Language and Environment for Statistical Computing. Available at: https://www.r-project.org (accessed July 5, 2019).

Rasoli, M., Yeap, S. K., Tan, S. W., Roohani, K., Kristeen-Teo, Y. W., Alitheen, N. B., et al. (2015). Differential modulation of immune response and cytokine profiles in the bursae and spleen of chickens infected with very virulent infectious bursal disease virus. BMC Vet. Res. 11:75. doi: 10.1186/s12917-015-0377-x

Ravel, A., Hurst, M., Petrica, N., David, J., Mutschall, S. K., Pintar, K., et al. (2017). Source attribution of human campylobacteriosis at the point of exposure by combining comparative exposure assessment and subtype comparison based on comparative genomic fingerprinting. PLoS One 12:e0183790. doi: 10.1371/ journal.pone. 0183790

Reid, W. D. K., Close, A. J., Humphrey, S., Chaloner, G., Lacharme-Lora, L., Rothwell, L., et al. (2016). Cytokine responses in birds challenged with the 
human food-borne pathogen Campylobacter jejuni implies a Th17 response. R. Soc. Open Sci. 3:150541. doi: 10.1098/rsos.150541

Richards, P. J., Connerton, P. L., and Connerton, I. F. (2019a). Phage biocontrol of Campylobacter jejuni in chickens does not produce collateral effects on the gut microbiota. Front. Microbiol. 10:476. doi: 10.3389/fmicb.2019.00476

Richards, P. J., Flaujac Lafontaine, G. M., Connerton, P. L., Liang, L., Asiani, K., Fish, N. M., et al. (2019b). Galacto-oligosaccharides modulate the juvenile gut microbiome and innate immunity to improve broiler chicken performance. bioRxiv[Preprint] doi: 10.1101/631259

Rosenquist, H., Nielsen, N. L., Sommer, H. M., Nørrung, B., and Christensen, B. B. (2003). Quantitative risk assessment of human campylobacteriosis associated with thermophilic Campylobacter species in chickens. Int. J. Food Microbiol. 83, 87-103. doi: 10.1016/S0168-1605(02)00317-3

RStudio Team (2015). RStudio: Integrated Development for R. Boston, MA: RStudio, Inc.

Rubio, L. A. (2019). Possibilities of early life programming in broiler chickens via intestinal microbiota modulation. Poult. Sci. 98, 695-706. doi: 10.3382/ps/ pey416

Sahin, O., Kassem, I. I., Shen, Z., Lin, J., Rajashekara, G., and Zhang, Q. (2015). Campylobacter in poultry: ecology and potential interventions. Avian Dis. 59, 185-200. doi: 10.1637/11072-032315-review

Schloss, P. D., Westcott, S. L., Ryabin, T., Hall, J. R., Hartmann, M., Hollister, E. B., et al. (2009). Introducing mothur: open-source, platform-independent, community-supported software for describing and comparing microbial communities. Appl. Environ. Microbiol. 75, 7537-7541. doi: 10.1128/AEM. 01541-09

Scott, A. E., Timms, A. R., Connerton, P. L., Carrillo, C. L., Radzum, K. A., and Connerton, I. F. (2007). Genome dynamics of Campylobacter jejuni in response to bacteriophage predation. PLoS Pathog. 3:e119. doi: 10.1371/journal.ppat. 0030119

Segata, N., Izard, J., Waldron, L., Gevers, D., Miropolsky, L., Garrett, W. S., et al. (2011). Metagenomic biomarker discovery and explanation. Genome Biol. 12:R60. doi: 10.1186/gb-2011-12-6-r60

Shaw, L. M., Blanchard, A., Chen, Q., An, X., Davies, P., Tötemeyer, S., et al. (2019). DirtyGenes: testing for significant changes in gene or bacterial population compositions from a small number of samples. Sci. Rep. 9:2373. doi: 10.1038/ s41598-019-38873-4

Silva, V. K., da Silva, J. D. T., Gravena, R. A., Marques, R. H., Hada, F. H., and de Moraes, V. M. B. (2010). Yeast extract and prebiotic in pre-initial phase diet for broiler chickens raised under different temperatures. Rev. Bras. Zootec. 39, 165-174. doi: 10.1590/S1516-35982010000100022

Smith, S., Messam, L. L. M., Meade, J., Gibbons, J., McGill, K., Bolton, D., et al. (2016). The impact of biosecurity and partial depopulation on Campylobacter prevalence in Irish broiler flocks with differing levels of hygiene and economic performance. Infect. Ecol. Epidemiol. 6:31454. doi: 10.3402/iee.v6.31454

Song, J., Xiao, K., Ke, Y. L., Jiao, L. F., Hu, C. H., Diao, Q. Y., et al. (2014). Effect of a probiotic mixture on intestinal microflora, morphology, and barrier integrity of broilers subjected to heat stress. Poult. Sci. 93, 581-588. doi: 10.3382/ps.201303455

Speksnijder, D. C., Mevius, D. J., Bruschke, C. J., and Wagenaar, J. A. (2015). Reduction of veterinary antimicrobial use in the Netherlands. The Dutch Success Model. Zoonoses Public Health 62, 79-87. doi: 10.1111/zph.12167

Swaggerty, C., Callaway, T., Kogut, M., Piva, A., and Grilli, E. (2019). Modulation of the immune response to improve health and reduce foodborne pathogens in poultry. Microorganisms 7:65. doi: 10.3390/microorganisms7030065

Tang, C., Kakuta, S., Shimizu, K., Kadoki, M., Kamiya, T., Shimazu, T., et al. (2018). Suppression of IL-17F, but not of IL-17A, provides protection against colitis by inducing Treg cells through modification of the intestinal microbiota. Nat. Immunol. 19, 755-765. doi: 10.1038/s41590-018-0134-y

Tang, K. L., Caffrey, N. P., Nóbrega, D. B., Cork, S. C., Ronksley, P. E., Barkema, H. W., et al. (2017). Restricting the use of antibiotics in food-producing animals and its associations with antibiotic resistance in food-producing animals and human beings: a systematic review and meta-analysis. Lancet Planet. Health 1 , e316-e327. doi: 10.1016/S2542-5196(17)30141-9

Thibodeau, A., Fravalo, P., Yergeau, É, Arsenault, J., Lahaye, L., and Letellier, A. (2015). Chicken caecal microbiome modifications induced by Campylobacter jejuni colonization and by a non-antibiotic feed additive. PLoS One 10:e0131978. doi: 10.1371/journal.pone.0131978

Thibodeau, A., Letellier, A., Yergeau, É, Larrivière-Gauthier, G., and Fravalo, P. (2017). Lack of evidence that selenium-yeast improves chicken health and modulates the caecal microbiota in the context of colonization by Campylobacter jejuni. Front. Microbiol. 8:451. doi: 10.3389/fmicb.2017. 00451

Tian, S., Wang, J., Yu, H., Wang, J., and Zhu, W. (2019). Changes in ileal microbial composition and microbial metabolism by an early-life galactooligosaccharides intervention in a neonatal porcine model. Nutrients 11:1753. doi: 10.3390/nu11081753

Van Bueren, A. L., Mulder, M., Van Leeuwen, S., and Dijkhuizen, L. (2017). Prebiotic galactooligosaccharides activate mucin and pectic galactan utilization pathways in the human gut symbiont Bacteroides thetaiotaomicron. Sci. Rep. 7:40478. doi: 10.1038/srep40478

Van Immerseel, F., Eeckhaut, V., Moore, R. J., Choct, M., and Ducatelle, R. (2017). Beneficial microbial signals from alternative feed ingredients: a way to improve sustainability of broiler production? Microb. sBiotechnol. 10, 1008-1011. doi: 10.1111/1751-7915.12794

Walliser, I., and Göbel, T. W. (2018). Chicken IL-17A is expressed in $\alpha \beta$ and $\gamma \delta \mathrm{T}$ cell subsets and binds to a receptor present on macrophages, and $\mathrm{T}$ cells. Dev. Comp. Immunol. 81, 44-53. doi: 10.1016/j.dci.2017. 11.004

Wang, H., Ni, X., Qing, X., Zeng, D., Luo, M., Liu, L., et al. (2017). Live probiotic Lactobacillus johnsonii BS15 promotes growth performance and lowers fat deposition by improving lipid metabolism, intestinal development, and gut microflora in broilers. Front. Microbiol. 8:1073. doi: 10.3389/fmicb.2017. 01073

Yang, X. O., Chang, S. H., Park, H., Nurieva, R., Shah, B., Acero, L., et al. (2008). Regulation of inflammatory responses by IL-17F. J. Exp. Med. 205, 1063-1075. doi: $10.1084 /$ jem. 20071978

Yoo, J., Yi, Y. J., Koo, B., Jung, S., Yoon, J. U., Kang, H. B., et al. (2016). Growth performance, intestinal morphology, and meat quality in relation to alpha-lipoic acid associated with vitamin $\mathrm{C}$ and $\mathrm{E}$ in broiler chickens under tropical conditions. Rev. Bras. Zootec. 45, 113-120. doi: 10.1590/S180692902016000300005

Yusrizal, and Chen, T. C. (2003). Effect of adding chicory fructans in feed on fecal and intestinal microflora and excreta volatile ammonia. Int. J. Poult. Sci. 2, 188-194. doi: 10.3923/ijps.2003.188.194

\section{Conflict of Interest: NF is employed by Dairy Crest Ltd.}

The remaining authors declare that the research was conducted in the absence of any conflict of interest.

The authors declare that this study received funding from Saputo Dairy UK. The funder had the following involvement with the study: NF contributed to the study design.

Copyright (c) 2020 Flaujac Lafontaine, Richards, Connerton, O'Kane, Ghaffar, Cummings, Fish and Connerton. This is an open-access article distributed under the terms of the Creative Commons Attribution License (CC BY). The use, distribution or reproduction in other forums is permitted, provided the original author(s) and the copyright owner(s) are credited and that the original publication in this journal is cited, in accordance with accepted academic practice. No use, distribution or reproduction is permitted which does not comply with these terms. 